\title{
Assessment of the Methodological Rigor of Case Studies in the Field of Management Accounting Published in Journals in Brazil
}

\author{
Kelly Cristina Mucio Marques \\ Adjunct Professor, Department of Accounting Sciences, State University of Maringá \\ E-mail: kcmmarques@uem.br \\ Reinaldo Rodrigues Camacho \\ Adjunct Professor, Department of Accounting Sciences, State University of Maringá \\ E-mail: rrcamacho@uem.br

\section{Caio Cesar Violin de Alcantara} \\ Undergraduate Student, Department of Accounțing Sciences, State University of Maringá \\ E-mail: caioalcantara_94@hotmail.com

\begin{abstract}
This study aims to assess the methodological rigor of case studies in management accounting published in Brazilian journals. The study is descriptive. The data were collected using documentary research and content analysis, and 180 papers published from 2008 to 2012 in accounting journals rated as A2, B1, and B2 that were classified as case studies were selected. Based on the literature, we established a set of 15 criteria that we expected to be identified (either explicitly or implicitly) in the case studies to classify those case studies as appropriate from the standpoint of methodological rigor. These criteria were partially met by the papers analyzed. The aspects less aligned with those proposed in the literature were the following: little emphasis on justifying the need to understand phenomena in context; lack of explanation of the reason for choosing the case study strategy; the predominant use of questions that do not enable deeper analysis; many studies based on only one source of evidence; little use of data and information triangulation; little emphasis on the data collection method; a high number of cases in which confusion between case study as a research strategy and as data collection method were detected; a low number of papers reporting the method of data analysis; few reports on a study's contributions; and a minority highlighting the issues requiring further research. In conclusion, the method used to apply case studies to management accounting must be improved because few studies showed rigorous application of the procedures that this strategy requires.
\end{abstract}

Keywords: case study, management accounting, research strategy. 


\section{INTRODUCTION}

In the 1980s, researchers understood little about the nature and determinants of management accounting practices (Scapens, 2004). The predominant research methods, including both surveys and statistical methods (Lee, Collier, \& Cullen, 2007), were initially used for that purpose. Scapens (1990) explains that surveys were conducted to find explanations about the nature of management accounting. However, the conclusions of survey studies were superficial, and therefore, case studies began to emerge, increasing interest in this research strategy.

Cooper and Morgan (2008) mention that the sensitivity of the context provided by the case study enables and encourages researchers to consider issues that may not be examined by other research approaches. Case studies in accounting may not find general solutions to the problems faced by managers and accountants, although they may provide a better understanding of the underlying issues (Scapens, 1990).

The case study is a research strategy that has generated development and discussions on some of its aspects (Berry \& Otley, 2004). For example, Otley and Berry (1994) argue that the specific contributions that case studies have provided have not always been clear.

As Yin (2010, p. 23) notes, "using case studies for research purposes remains one of the most challenging of all social science endeavors"; Yin also emphasizes that this type of research is difficult, although conventionally considered soft, perhaps because researchers using the case-study approach do not follow systematic procedures. Godoy (2006) states that the case study is commonly considered a strategy that allows some flexibility, but specific epistemological principles and methodological procedures must be followed and respected to develop quality work.

The case study rationale is based on the interpretation of the data and evidence of real social phenomena. Therefore, several processes are essential to the validity and reliability of its results; these aspects are discussed by various authors, including both Eisenhardt (1989) and Yin (2010). Thus, the case study strategy demands skill and sensitivity from the researcher to ensure that these requirements are met so that the results of the study are accompanied by methodological rigor and accepted in the scientific community.

Martins (2006) highlights that the research strategy guided by a case study in many applied social sciences studies has not been adopted according to what is expected from a scientific study. Yin (2010) states that perhaps the greatest concern about the case study is its lack of rigor. Alves-Mazzotti (2006) argues that the problem with most studies that are published as case studies is that they are not case studies. Cepeda and Martin (2005) argue that both the increased use of case studies in the management field and the desire to construct theories based on qualitative data have shown a gap between the existing proposals and the criteria for the development of rigorous case studies.

A study conducted by Cesar, Antunes, and Vidal (2010) in the field of accounting concludes that the case study method has been used without methodological rigor, especially in Brazil. That conclusion raises the question of how to use the case study strategy in management accounting, that is, how are issues regarding methodological rigor considered in published management-accounting papers in Brazil?

Because of its importance and the issues raised above, this study aims to examine the methodological rigor of published management-accounting papers in Brazil that use case studies.

Cesar et al. (2010) and Cesar, Antunes, and Vidal (2008) analyze the case study strategy in scientific production in the field of accounting in Brazil. Consoli, Musetti, Scare, and Fratantonio (2008) and Gil, Licht, and Oliva (2005) review the use of case study in the field of management. However, no papers evaluating management accounting have been found.

Another issue is that these studies found evaluated studies published in meeting reports, whereas this study's analysis focuses on journals, because they publish mature studies on research that either is already completed or has been discussed in meetings (Cesar et al., 2010). This study was performed in Brazil because the Brazilian context with respect to the use of the case study strategy is unknown. Therefore, it is appropriate to conduct a study whose purpose is to gather information on case studies conducted in the country, showing which points have been met and which have been neglected by researchers.

This study aims to contribute information about the use of the case study, focusing on methodological rigor and providing evidence about how the strategy has been used. This is important because as previously indicated, there are problems related to the use of the case study strategy that must be identified and discussed by the scientific community to improve its use. Another contribution is the description of a set of guidelines for conducting case studies; in addition, we make suggestions aimed at supporting the search for methodological rigor, which are provided throughout the text. This study's findings may guide efforts to improve the methodological rigor of future studies that will use the case study as a research strategy, resulting in greater reliability and validity for the results of studies published in Brazil. 


\section{LITERATURE REVIEW}

\subsection{Case Study Definition and Characteristics.}

The definition, use, and ontological and epistemological aspects of the case study are not unanimously agreed upon by scholars. Explicit definitions of the case study are not very fruitful without considering the context of its use and the user's perspective because this approach refers to the methodological needs of different scientific schools of thought or perspectives (Hägg and Hedlund, 1979).

Case studies are differentially understood depending on those needs. Scapens (1990) indicates that the use of case study methods depends both on the type of research and the methodology of the researcher. The author noticeably refers to case study methods instead of method, suggesting that the term may be understood and applied differently by different people. In general terms, the case study is a type of field research that seeks to understand the reality of a given topic by focusing on one or several units of analysis. Some definitions of case study are introduced and discussed below.

The case study is a research strategy focused on understanding the dynamics present within a single setting (Eisenhardt, 1989). Understanding these dynamics involves understanding what is being done and what the dynamics mean. Another aspect of this definition is the term "a single setting", which is understood as a unit of analysis, that is, the phenomenon or subject examined, which may be a company, person, department, method or event, among phenomena and subjects.

The case study is performed through empirical research that engages in an in-depth examination of a contemporary phenomenon and its real-life context, especially when the boundaries between phenomenon and context are not evident (Yin, 2010). Pursuant to this definition, the author focuses on the scope of the case study and addresses the real-life context, stressing that the researcher does not separate the phenomenon from its context because contextual conditions are important for its understanding. The goal is to understand or comprehend the phenomenon, not to establish causal relationships.

In the second part of his definition, Yin (2010) notes that an unclear boundary between phenomenon and context is often indicative of the use of the case study. However, this aspect requires further details, such as the establishment of other technical characteristics, including data collection and data analysis strategies, to narrow the definition of case study. Thus, Yin (2010) argues that the case study is a broad method that includes project rationale, data collection methods, and specific approaches to data analysis approaches. This is a noteworthy issue because, according to Martins (2008), the case study is a research strategy and not a data collection method; that is, the mere conduct of research using data from a company does not render that research a case study.
According to Yin (2010), research involves a technically different situation wherein there will be many more variables of interest than there are data points. Therefore, research relies on multiple sources of evidence and data, which should converge triangularly, thereby benefiting from the prior development of theoretical propositions to guide data collection and data analysis.

With respect to the case study methodology, Yin (2010) comments that the research result is related to the theoretical propositions used as its basis, that is, the researcher begins the field research armed with propositions or hypotheses drawn from a basic theory. Conversely, Merriam (2009) advocates the use of case study when the researcher's interest is more focused on understanding the social processes that occur in a specific context than on the relationships established between variables. Furthermore, the author also notes that the case study focuses more on insight, discovery, and interpretation than on hypothesis testing. In this type of study, the researcher goes into the field to find out what happens, seeking to understand how things work, which is a process that is both intuitive and inductive.

This study does not intend to discuss which of the case study approaches is most appropriate; instead, it understands that the choice of one or the other depends on the purpose of the study, the type of question that will be researched, and the type of analysis that will be extracted. Thus, a deductive analysis considers a theoretical framework as the premise, and an inductive analysis is more dependent on interpretation and intuition about the data, analyzed separately.

Meer-Kooistra and Vosselman (2012) argue that the search for more practically relevant management accounting knowledge is related to the debate on the desirability of theoretical pluralism or heterogeneity in the production of that knowledge. According to Cesar et al. (2010), the case study may have a positivist, a post-positivist, or a post-structuralist approach, among others; the approach will affect not the choice of method but instead the element chosen for analysis, the method of data selection and analysis, and the language used to report the case.

According to Yin (2010), the case study is interesting when it fulfills three requirements, namely: (i) the questions posed are "how" or "why"-type questions; (ii) the researcher has little control over the events; and (iii) the focus is on a contemporary, real-life phenomenon. Godoy (2006) adds that comprehension-type questions that seek to describe and interpret "what" happened in a specific situation are also important. Cepeda and Martin (2005) argue that some reasons to use the case study include the opportunity for the researcher to study management in its natural environment, to learn about its current status, and to generate theories from practice; to answer "how" and "why"-type questions to understand the nature and complexity of processes; and to explore areas in which research is scarce. 
One inherent characteristic of the case study is its deepening capacity for data, analysis, and results compared to other research strategies. Lillis and Mundy (2005) have conducted a study on the research strategies used in management accounting studies, illustrating the levels of depth and breadth (high and low) into which strategies may be classified (Figure 1). Case studies (single and multiple) are placed at the high level of depth and low level of the breadth category because of the size of the samples analyzed.

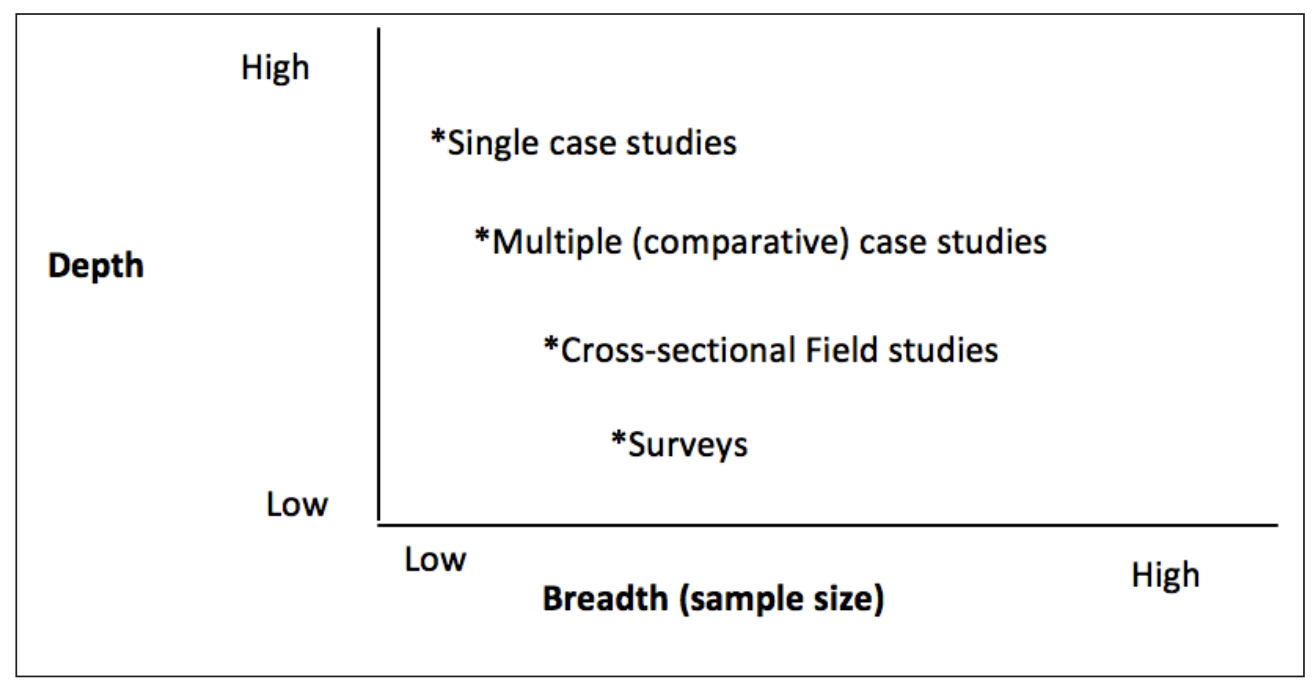

Source: Adapted from Lillis and Mundy (2005, p. 132).

Figure 1 Comparison between depth and breadth of research strategies.

Thus, questions that require deepening the resear$\mathrm{ch}$ on the subject or phenomenon being studied use the case study strategy.

\subsection{Types of Case Study.}

Case studies, similar to other research strategies, may be used for various purposes. Yin (2010) argues that case studies may be exploratory, descriptive, and explanatory, and each should be classified according to the type of question. However, the author provides no further information about what characterizes each type of case study.

Lee et al. (2007) remark that exploratory case studies tend to be conducted as preliminary research before large-scale surveys to identify topics for further research. Descriptive case studies are commonly used to expand the trends and topics that have already been discovered by surveys. Only the explanatory case seeks to extract a detailed understanding of a particular phenomenon when the case is not regarded as an accessory to quantitative methods.

Eisenhardt (1989) establishes a different classification in which case studies may be used for several purposes, including providing a description, testing a theory, or generating a theory. Cesar et al. (2010) follow that line of reasoning to define the objectives pursued by researchers to apply the case study and argue that defining those objectives will help define the knowledge generated by the study.

Scapens (1990) suggests that management accounting case studies may be descriptive, illustrative, experimental, exploratory, or explanatory. Descriptive case studies des- cribe the accounting systems, methods, and procedures currently used in practice. Illustrative case studies show new and possibly innovative practices developed by specific companies. Experimental case studies are used to examine the difficulties of implementing new proposals and to evaluate the benefits derived from them. Exploratory case studies are preliminary investigations aimed at generating ideas and hypotheses for rigorous empirical testing at a later stage, with the purpose of promoting generalizations about the reasons for various accounting practices. Explanatory or explicative case studies seek to explain the reasons for accounting practices wherein a theory is used to understand and explain specific objectives rather than to produce generalizations.

These classifications show that the type of case study will depend on the objective and the knowledge on a particular subject in relation to that contained in the literature. For example, a study that aims to assess how a specific accounting practice works in a company will use the descriptive type of case study, whereas another study aiming to identify patterns of behavior regarding specific decisions among a group of managers in an organization to formulate a substantive theory based on the data will use the explanatory type, and another seeking to clarify a practice used in a company will use the exploratory type.

\subsection{Criteria for Case Study Quality Assessment.}

Four tests have been used to assess the quality of empirical social research studies: construct validity, internal validity, external validity, and reliability (Yin, 2010). 
The first three tests refer to validity, and the last test refers to reliability. Validity concerns the instrument's ability to measure the object of the subject, whereas reliability is related to the consistency of the results obtained when the same individual or subject is evaluated, quantified, or measured more than once (Martins \& Theóphilo, 2007).

Thus, construct validity is used to assess whether the operational definitions and constructs actually measure the study object. Yin (2010) notes that the operational measures appropriate for the study concepts should be identified. Internal validity only concerns the explanatory case study type and refers to the quest to establish the causal relationship whereby specific conditions presumably lead to other conditions, different from spurious relationships (Yin, 2010). Godoy (2006) presents a different interpretation, wherein the findings of a research study must be based on data to be internally valid, that is, the extent to which the description of the case represents the data collected.

Internal validity is related to the data analysis phase, and its assessment consists of ensuring that the analysis results are supported by logical models of argument development and simultaneously ensuring that they have the ability to reflect the reality studied.

External validity defines the domain to which the study findings may be generalized (Yin, 2010), including some actions that enable some type of generalization of the results. Clearly, this is not statistical generalization (inference by sampling) but instead a type of generalization that Yin (2010) calls analytical generalization, which is linked to a broad theory.

The issue of generalizing case study results is a recurring subject because this strategy addresses specific realities, not samples of realities. Scapens (1990) clarifies the issue, showing that case studies are approached as small-sample studies, and this interpretation, at least in management accounting, derives from the positive research tradition, which aims to determine the extent of particular occurrences in a specific population.

Yin (2010) also follows this line of reasoning when commenting on this type of criticism, mentioning that it occurs when comparing the case study to the survey. However, this analogy with samplings and universes is incorrect when examining case studies because surveys rely on statistical generalization, whereas case studies rely on analytical generalization.

Thus, when compared to strategies based on quantitative models, the case study has this limitation. However, when the analysis is focused on the nature and purpose of the case study, this issue loses relevance because the objective of the case study is not the statistical generalization of a specific phenomenon but instead the understanding of a specific phenomenon as it actually occurs, regardless of whether other similar situations occur.

Reliability relates to the demonstration that the operations of a study, including data collection procedu- res, may be repeated with the same results (Yin, 2010). Reliability ensures the possibility of replicating a study, and the researcher should implicitly follow specific procedures inherent to scientific research. Because a case is usually non-replicable, its reliability will be primarily demonstrated by data triangulation resulting from the use of various data collection tools, evidence linking, and rigor in all of the procedures performed during the study (Martins, 2006). Cesar et al. (2010) assesses the existence of data triangulation as one of the points of evaluation of methodological rigor.

Yin (2010) suggests the use of a case study protocol to ensure that the study or one of its procedures may be repeated. The protocol consists of detailed documentation of the procedures followed in the study so that those records increase the reliability of the method.

Another noteworthy issue for researchers is the importance of highlighting the knowledge generated by the case study (Otley and Berry, 1994). Cesar et al. (2010) also discuss this characteristic, specifying some criteria related to the finding or knowledge generated by the case study. Accordingly, the case study's importance lies in identifying not only the findings or knowledge that it generates for theory or practice but also whether grounds for further studies exist.

Alves-Mazzotti (2006) argues that the validation of the knowledge generated by the case study and the approval of its reliability and relevance by the academic community require the researcher to be acquainted with the current status of knowledge on the study subject, enabling researchers (in some way) to include their research in the collective knowledge-production process.

According to the above, the case study strategy has requirements to ensure that it is truly scientific through valid and reliable results, like any other strategy, and the researcher must be aware of these issues.

\subsection{Roadmap for Conducting a Case Study.}

It is important to note that the establishment of procedural guidelines, especially to produce a research strategy, is not a package deal that ensures a study's success. The objective is to establish some steps and procedures that will help in planning and conducting the study, if observed by the researcher. There is no unanimity among authors about the roadmap, although those who care about the issue suggest some steps to help conduct a case study.

Godoy (2006) discusses the choice of the unit of analysis, the definition of the role of theory, the literature review, and the data collection and data analysis procedures. The case study type will depend on the study subject, which will determine how the other steps are defined. The unit of analysis corresponds to the site at which the study will be conducted. Furthermore, when, who, what, how and where to observe should also be assessed, according to Godoy (2006). Whether the study will be a single- or multiple-case study is also defined at this step. 
According to Yin (2010), when designing a case study, it is important to decide whether the research questions will be approached using a single case or multiple cases. The single-case study is appropriate in several circumstances and Yin (2010) presents five such circumstances: (i) when the case is critical to testing a theory and can confirm, challenge, or expand it; (ii) when the case is extreme or peculiar; (iii) in contrast to the previous explanation, when the case is representative or typical; (iv) when the case is revealing and previously inaccessible to social science research; and (v) when the case is longitudinal, representing two or more time points.

Conversely, the choice to perform a multiple-case study is explained by the need for literal (cases may predict similar results) and theoretical (produce contrasting results, but for predictable reasons) replications. In other words, the rationale for the use of multiple cases is replication (Yin, 2010).

The type of case study that will be conducted should consider the role of theory. As discussed above, the use of a certain theory is not mandatory, except when the objective is to test it or when a theory is used to establish propositions to guide the study.

Literature review is regarded as relevant in contributing to the advancement of knowledge because existing studies on the subject should be considered, preventing the study from being trivial or addressing an area that is already well studied (Godoy, 2006). Otley and Berry (1994) argue that the case study is not theory-free; it begins with some implicit or explicit theoretical stance. The importance of theory choice or even of the review used to guide the case study results from the possibility to show the study contribution, that is, to identify the study result that remains unknown to the academic or practice community.

Case studies combine data collection methods such as interviews, records, questionnaires, verbal reports, and observations; the evidence may be qualitative and quantitative (Eisenhardt, 1989; Yin, 1981), or it may be qualitative only (Godoy, 2006). Cesar et al. (2010) assess whether the data collection methods used were explained and the existence of explanations of the collection method, including both the number of respondents and the type of data collected.

Data analysis should be surrounded by the logical sequence linked to the type of question and the type of study. The analysis should be confined to the data in the case of an inductive study, that is, it should describe what the data show, in the sense of describing a reality or generating a theory. In the data analysis of deductive studies, the theory that serves as the basis to formulate the proposals and guide the study should be considered.

Scapens (1990) considers the preparation, collection of evidence, evaluation of evidence, identification and explanation of the standards, the development of the theory, and the writing of the report as key steps that should be followed when conducting a case study. There are some differences with respect to what Godoy (2006) proposes, although in essence, the same logic is followed.

Yin (2010) considers these issues as components of the case study design, including the study questions; the propositions, when present; the unit of analysis; the rationale linking the data to the propositions; and the criteria to interpret the findings. Lima, Antunes, Mendonça Neto, and Peleias (2012) propose a theoretical framework for the development and validation of the case study according to the following steps: (i) formulation of the problem; (ii) definition of the case unit; (iii) determination of the number of cases; (iv) protocol development; (v) data collection; (vi) evaluation, analysis, and triangulation of data; and (vii) report preparation.

These roadmaps are important so that researchers know in advance the steps and procedures to be followed to ensure that the research project addresses important concerns. It is important to note that these steps are included in the criteria for assessing the methodological rigor of the present study.

\subsection{Previous Studies Conducted in Brazil.}

Cesar et al. (2010) assess the methodological rigor of papers published in the Brazilian Academy of Management (Associação Nacional de Pós-Graduação e Pesquisa em Administração - ANPAD) Meeting and in the Journal of Accounting Research, which used the case study. They developed a model with sixteen criteria. The conclusions highlighted that the case study strategy has been used without methodological rigor, especially in Brazil.

Gil et al. (2005) analyze case studies from a meeting in the field of management; the most critical aspects are the following: the use of few sources of evidence in most studies; the lack of clarity in the analytical procedures; and the fact that few studies were characterized by the application of rigorous procedures.

Consoli et al. (2008) analyzes the case study in meetings in the management and production engineering fields; his results find a predominance of studies with descriptive and exploratory purposes with comparative and linear structures; few studies that aimed to propose theories and models; a lack of methodological rigor and planning when conducting case studies with respect to case selection, collection instruments, data analysis, and conclusions.

\section{METHODOLOGICAL PROCEDURES}

This study is descriptive because it sought to describe characteristics of a population by examining the metho- dological rigor of case studies in management accounting published in Brazil. 


\subsection{Population and Sample.}

Papers that were published in Brazilian accounting journals ranked in strata A2, B1, and B2 of the Coordination for the Improvement of Higher Education Personnel (Coordenação de Aperfeiçoamento de Pessoal de Nível Superior, Capes) Quality were selected. Those strata were chosen because they have the highest Capes scores in Brazil. The period analyzed ranges from 2008 to 2012 because most of the academic master's and doctorate degrees in accounting sciences began to be granted in 2007, which may have contributed to the production and publication of research studies in the country.

Data collection was performed in May 2013; we selected those management accounting papers that used the research strategy of the case study. The term "case study" was searched in the paper title or abstract, or in the body of the text when necessary, to identify and select the papers for analysis. Importantly, only the papers whose authors clearly indicated that it was a case study were selected.
Subsequently, the term "management accounting" was searched in the abstracts and in some cases, in the full text of the papers. Whenever this term was not explicitly indicated, the paper introduction was read to assess whether the study focused on a subject related to the field of management accounting. For example, papers on management information systems, information for decision-making, and management accounting practices and artifacts were found. Those that did not include the term or that were not focused on a subject of the field were excluded from the sample.

A total of 181 papers were identified and selected for analysis. However, the full-text of one paper was not available online, and therefore, it was excluded from the sample. The 180 papers reviewed are listed by journal in Table 1. 28 papers published in 2008, 40 published in 2009, 44 published in 2010, 35 published in 2011, and 33 published in 2012 were evaluated. The journals classified in the strata studied that are not presented in Table 1 published no papers on the subject examined in the period covered by the study.

Table 1 List of journals used in the research study and the number of sample papers

\begin{tabular}{|c|c|c|c|c|}
\hline & Stratum & ISSN & Journal & Number \\
\hline 1 & $\mathrm{~A} 2$ & $1519-7077$ & Revista de Contabilidade e Finanças & 5 \\
\hline 3 & B1 & 0103-734X & Contabilidade Vista e Revista & 11 \\
\hline 4 & B1 & $1982-6486$ & Revista Contabilidade e Organizações & 10 \\
\hline 5 & B1 & 1809-3337 & Revista Universo Contábil & 18 \\
\hline 6 & B1 & 1984-8196 & Base (São Leopoldo - Online) & 24 \\
\hline 7 & B1 & 1983-0807 & Revista Brasileira de Gestão de Negócios & 3 \\
\hline 8 & B2 & $1983-8611$ & Advances in Scientific and Applied Accounting & 3 \\
\hline 9 & B2 & $1984-3925$ & Contabilidade, Gestão e Governança & 13 \\
\hline 10 & B2 & 1678-2089 & Contextus & 7 \\
\hline 11 & B2 & $1808-2882$ & Custos e Agronegócios & 53 \\
\hline 12 & B2 & $1984-882 X$ & Enfoque: Reflexão Contábil & 12 \\
\hline 13 & B2 & $2176-9036$ & Revista Ambiente Contábil & 5 \\
\hline 14 & B2 & $1981-8610$ & Revista de Educação e Pesquisa em Contabilidade & 3 \\
\hline \multirow[t]{2}{*}{15} & B2 & $2179-4936$ & Revista de Administração, Contabilidade e Economia & 2 \\
\hline & & & Total & 180 \\
\hline
\end{tabular}

\subsection{Criteria Providing Methodological Rigor to the Case Study Based on the Literature.}

A set of 15 criteria (Table 2), whose explicit or implicit inclusion in case studies is desirable for the studies to be considered appropriate from the standpoint of methodological rigor, were established based on the issues discussed in the literature review section (theoretical framework). More specifically, this study was based on Cesar et al. (2010), with six criteria adapted from that study. The other criteria were extracted from other studies composing the theoretical framework because they were deemed more appropriate as methodological rigor measures. The criteria were classified into four categories: (i) the study object; (ii) the data collection/ evidence; (iii) the data analysis; and (iv) the results. 
Table 2

Criteria for the analysis of methodological rigor in case studies

\begin{tabular}{|c|c|c|c|}
\hline Categories & & Criteria & Authors \\
\hline \multirow{6}{*}{ The study object } & 1 & $\begin{array}{l}\text { Does the study seek to understand a phenomenon in its real-life context? (explanation } \\
\text { of the need to use the case study to examine the proposed phenomenon, which is not } \\
\text { possible through other strategies) }\end{array}$ & $\begin{array}{l}\text { Yin (2010); Eisenhardt (1989), } \\
\text { Cepeda and Martin (2005) }\end{array}$ \\
\hline & 2 & $\begin{array}{l}\text { Was the reason for the choice of this strategy explained? (to test theories, construct } \\
\text { theories, or describe a phenomenon or explore the phenomenon, among others) }\end{array}$ & $\begin{array}{l}\text { Yin (2010); Eisenhardt (1989); } \\
\text { Scapens (1990); Cesar et al. } \\
\text { (2010) }\end{array}$ \\
\hline & 3 & $\begin{array}{l}\text { Is there a connection between the phenomenon and the context at some research } \\
\text { stage? (need to understand the phenomenon in that context) }\end{array}$ & Yin (2010) \\
\hline & 4 & What is the type of question raised in the study? (how, why, what) & $\begin{array}{l}\text { Yin (2010); Godoy (2006), } \\
\text { Cepeda and Martin (2005) }\end{array}$ \\
\hline & 5 & What is the case study type? (exploratory, descriptive, explanatory, among others) & $\begin{array}{l}\text { Yin (2010); Scapens (1990); } \\
\text { Eisenhardt (1989) }\end{array}$ \\
\hline & 6 & $\begin{array}{l}\text { Is the case analyzed representative of the study objective? (discloses explanations for } \\
\text { the choice of a single-case or multiple-case study) }\end{array}$ & Godoy (2006); Yin (2010) \\
\hline \multirow{5}{*}{ The data collection } & 7 & $\begin{array}{l}\text { Are there multiple sources of evidence? (interviews, observation, examination of } \\
\text { documents, among others, to enable the triangulation of data) }\end{array}$ & $\begin{array}{l}\text { Eisenhardt (1989), Yin (1981), } \\
\text { Godoy (2006), Cesar et al. (2010) }\end{array}$ \\
\hline & 8 & $\begin{array}{l}\text { Is there triangulation of data between the sources of evidence? (reliability } \\
\text { characteristic) }\end{array}$ & $\begin{array}{l}\text { Yin (2010); Martins (2008); Lima } \\
\text { et al. (2012), Cesar et al. (2010) }\end{array}$ \\
\hline & 9 & $\begin{array}{l}\text { Were operational measures regarding the variables analyzed disclosed, when } \\
\text { necessary? (construct validity) }\end{array}$ & Yin (2010) \\
\hline & 10 & $\begin{array}{l}\text { Is there an explanation for the data collection method, including the steps followed, } \\
\text { when they occurred, where they occurred, with whom, and in what way? (reliability } \\
\text { characteristic) }\end{array}$ & Yin (2010), Cesar et al. (2010) \\
\hline & 11 & $\begin{array}{l}\text { Is there any report or disclosure regarding the research protocol? (possibility of data } \\
\text { collection replication) }\end{array}$ & Yin (2010) \\
\hline \multirow[t]{2}{*}{ The data analysis } & 12 & $\begin{array}{l}\text { Is there an explanation for the method of analysis? (internal validity: do the results } \\
\text { express the data? or are the results of the analysis based on logical models of } \\
\text { development of arguments?) }\end{array}$ & Godoy (2006); Yin (2010) \\
\hline & 13 & $\begin{array}{l}\text { Were theory (single-case study) or replication (multiple-case study) used as a basis for } \\
\text { the analysis when conducting a deductive study? (external validity characteristic) }\end{array}$ & $\begin{array}{l}\text { Yin (2010), Otley and Berry } \\
\text { (1994) }\end{array}$ \\
\hline \multirow[t]{2}{*}{ The results } & 14 & $\begin{array}{l}\text { Were contributions to knowledge generation reported in comparison to previous } \\
\text { studies? }\end{array}$ & $\begin{array}{l}\text { Cesar et al. (2010), Otley and } \\
\text { Berry (1994) }\end{array}$ \\
\hline & 15 & Does the study warn about issues requiring further research? & Cesar et al. (2010) \\
\hline
\end{tabular}

The criteria formed the matrix guiding the data analysis in the present study.

\subsection{Procedures for Data Collection and Analysis.}

Methodological rigor was understood as the frequency (or the presence) of aspects characteristic of the case study strategy outlined in Table 2 in each paper in the sample examined. The value one (1) was given when the characteristic was found in the text. The value zero (0) was given when the paper failed to show the characteristic. In the case of multiple-response criteria (including items 4,5 , and 13), the type of each response was described and the frequencies of similar responses were then added. The 180 papers analyzed were evaluated by reading each paper, especially the introduction, metho$\mathrm{ds}$, and conclusion. In some cases, it was necessary to read the presentation and analysis of results to assess some items.

The method applied by Cesar et al. (2010) was used for the data analysis, wherein each criterion was analyzed in each paper so that criterion 1, for example, will have $100 \%$ methodological rigor if all of the sampled papers contain that criterion. Thus, the closer to $100 \%$ the criterion is, the higher the methodological rigor it will show in the sample. The objective was not to assign a cut-off point to highlight which criteria had high or low methodological rigor; instead, according to the analysis performed, the criteria less aligned with the theoretical propositions and those under observation by the authors when performing the case study could be identified.

One limitation of this study relates to the choice of the criteria used to evaluate papers; those criteria are focused on issues such as construct validity, internal validity, external validity, and reliability, which are test models applied in case studies with a positivist approach, according to Cepeda and Martin (2005). This approach is part of the accounting mainstream, according to Chua (1986), and was adopted because nearly all of the papers found for analysis did so. Although discussing or analyzing the approaches used in the papers was not the objective of this 
study, an initial assessment was performed for the criteria to be chosen. Only two papers ( $1 \%$ of the total) reported using the interpretive research approach. Therefore, the positivist approach criteria were adopted, focusing on Yin (2010), Scapens (1990), and Eisenhardt (1989).
Another limitation is the impossibility of extrapolating the results found beyond the sample analyzed because the sample is non-probabilistic. Therefore, the results found are valid for the period and journals analyzed and may not be used for generalizations related to other journals or periods.

\section{PRESENTATION AND ANALYSIS OF RESULTS}

\subsection{Methodological Rigor Assessment.}

Criteria 1 to 6 analyzed the papers in terms of their study objects. Criterion 1 assessed whether the study sought to understand the phenomenon in its real context, that is, the existence of an explanation in each paper about the need to use the case study to examine the proposed phenomenon, which was not possible using other research strategies. Only $22 \%$ of the sample (39 papers) contained an explanation emphasizing the importance of choosing the case study. This information indicates that for most authors, the choice of this research strategy does not require further clarification or discussions of its suitability.

An explanation for this percentage may be that the authors did not consider it important to explain the choice of research strategy used in their studies. The information that the study will be conducted in a specific company in the paper introduction, for example, may convey the incorrect idea that this issue is being covered without providing further information about why the strategy was chosen. That is, confusion may exist between defining the study site and explaining the choice of research strategy. This is a key issue for research quality because the case should express the reality that the study seeks to understand, as indicated by Yin (2010), Cepeda and Martin (2005), Cooper and Morgan (2008), and Scapens (2004).

Criterion 2 assessed whether the reason for choosing the case study strategy was explained, that is, to test or develop theories, or to describe or explore a phenomenon, among others. This information was reported in 23 papers (13\%). This result differs from Cesar et al. (2010), who find $100 \%$ frequency of this criterion. That compliance was not surprising to Cesar et al. (2010), which is explained by the fact that one criterion considered by journals to accept papers is the existence of clearly defined objectives for the application of the strategy. However, the research sample of those authors consisted of papers published in conference annals rather than journals. The $13 \%$ frequency in the case of this study contradicts that explanation because although most papers failed to report clear objectives, they were published by journals.

Thus, a possible explanation for this result may be reviewers and journals' failure to require reporting of the papers' objectives. Another explanation may be linked to the failure of authors to consider reporting this type of information as important (or even their ignorance of this need). The importance of this criterion is highlighted because this information outlines the study scope and guides the research methodology and findings. Otley and Berry (1994) and Ahrens and Chapman (2006) describe the importance of emphasizing this choice, which helps to show the research findings in relation to other studies, which affect another criterion, the reporting of the study contribution, which is analyzed below.

Criterion 3 assessed whether a link existed between the phenomenon and the context at any stage of the research, that is, whether understanding the phenomenon is necessary in that context. This information was found in 149 papers $(83 \%)$ and was more frequently reported in the introduction and in some cases, in the methods. Although Cesar et al. (2010) do not make an independent assessment of this characteristic, the combined analysis of the studied phenomenon showed that authors are not significantly committed to reporting the criteria used for case selection and that one explanation for this is the difficulty in accessing companies, which ultimately occurs not by necessity, but for reasons of convenience.

Although convenience may be one of the reasons to conduct a case study at a specific company, that does not explain the failure to report the reasons for doing so. After all, whether it is suitable or not, the study will ultimately be conducted at that company, and thus, there should be some reason that explains the choice. Otherwise, the study will not generate contributions because it is not performed in a suitable environment. In this study, the presence of explanations in $83 \%$ of the sample suggests the authors were concerned about explaining the importance of performing the case study in a particular company or environment and explained their choice. This may suggest that researchers have no difficulties in finding a suitable site to conduct their studies.

Criterion 4 assessed the type of question raised in the study. Questions beginning with "how" appeared in 25 papers, "which"-type questions appeared in 35 papers, questions with "yes/no" answers appeared in 22 papers, other types of questions appeared in three papers, and 113 papers showed no question whatsoever. The frequency could not be assessed in this case because many papers contained two or more questions.

The case study method is preferable for "how" and "why"-type questions, according to Yin (2010), as discussed above. Most of the papers analyzed cite this statement by Yin but most do not use those types of questions to 
guide the case study. Furthermore, the use of which-type questions and questions with yes/no-type answers precludes a deeper analysis of the results. It is also noteworthy that 113 papers (63\%) do not report questions of any type. One reason that most papers present no questions, "which"-type questions, or questions with "yes/no"-type answers may be that most studies were descriptive or exploratory, according to criterion 5 discussed below, which usually have no intent to explain the findings.

Criterion 5 examined the type of case study - whether exploratory, descriptive, or explanatory, among others. Most fall into the exploratory (25\%), descriptive (23\%), and exploratory/descriptive (12\%) categories, according to Table 3. The analyses showed that a deeper data analysis was rarely seen in these types of studies. In the sample analyzed, these types of case study compared the data found with the literature reviewed but did not discuss the possible causes of the differences or similarities found in the sample analyzed. Otley and Berry (1994) mention that a core function of the case study is to explore, although the central idea of the exploratory study surpasses mere description and is guided towards explanation. This strengthens the notion that the manner in which exploratory case studies were used in the papers makes little sense because the in-depth study of a phenomenon is one of its objectives.

Consoli et al. (2008) also find a high number of case studies with descriptive and exploratory purposes. This panorama may suggest that researchers are not deepening their understanding or spending the time necessary in the early stages of the research, including in the planning of what to do and how to do it. Furthermore, the need or even the preference for conducting studies that demand less time to complete may help explain the choice of the types found. According to Martins (2008), the issue of time limits the range of research possibilities guided by a case study.

It is noteworthy that the type of research was not mentioned in 62 papers (34\%). This information helps to outline what is expected from the case study results and is included in the research plan and should be explained to the reader to facilitate understanding the scope of the results found in the study.

\section{Table 3}

\begin{tabular}{lcc}
\hline Criterion 5 & Total & \% \\
\hline Exploratory & 45 & $25 \%$ \\
\hline Exploratory and descriptive & 22 & $12 \%$ \\
\hline Not mentioned & 62 & $34 \%$ \\
\hline Interpretive and explicative & 1 & $1 \%$ \\
\hline Descriptive & 41 & $23 \%$ \\
\hline Action research & 1 & $1 \%$ \\
\hline Explanatory, descriptive, and exploratory & 1 & $1 \%$ \\
\hline Interpretive & 1 & $1 \%$ \\
\hline Descriptive and explicative & 1 & $1 \%$ \\
\hline Explicative & 3 & $2 \%$ \\
\hline Illustrative & 1 & $1 \%$ \\
\hline Explicative and exploratory & 1 & $1 \%$ \\
\hline Total & $\mathbf{1 8 0}$ & $\mathbf{1 0 0} \%$ \\
\hline
\end{tabular}

Table 3 also shows that the explicative or explanatory type appears in only $6 \%$ of cases and is sometimes used in combination with other types of research. Otley and Berry (1994) highlight that case studies are most likely used to help generate theoretical statements through observations and descriptions, which was not found in the sample. Consoli et al. (2008) also find few examples of research studies for the development and proposition of theories and models based on case studies. The presentation of one of the papers as an action research-type case study, which according to Martins and Theóphilo (2007) is another research strategy, was interesting and shows the lack of understanding of the strategy adopted.

Criterion 6 assessed whether the case analyzed was representative of the study objective, that is, whether the study presented explanations for the choice of a single case or multiple cases. This explanation was observed in 51 papers $(28 \%)$. Importantly, the analyses could not be separated between single cases and multiple cases because in some papers, the studies were conducted in several companies. However, they were considered single-sector case studies, not multiple-case studies. It was also found that some multiple-case studies applied the same method but did not discuss the reproducibility or comparison of results. Others also examined several companies or units of analysis and failed to mention whether it was a multiple-case or a single-case study.

The explanation for the unit of analysis and the number of cases examined is important because it provides evidence on the choice of the case, its representativeness of the study subject, and the factors used for the choice, as indicated by Yin (2010). The fact that only $13 \%$ of authors reported explanations for the choice of the case study strategy, according to criterion 2, may help explain the frequency found, that is, the apparent lack of concern about explaining the case choice.

Criteria 7 through 11 analyzed the studies with respect to their data collection. Criterion 7 assessed whether the studies contained multiple sources of evidence to enable data triangulation. More than one source of evidence was used in 105 papers (58\%). Although that number represents the majority, $42 \%$ of case studies are were based on only one source of data, which precludes confirming the accuracy of the information used in the analyses, that is, it precludes triangulating the data and ensuring data reliability. Cesar et al. (2010) do not evaluate the type of evidence source, although they find that $95 \%$ of the papers evaluated described the data collection methods.

According to Gil et al. (2005, p. 50), "One of the most distinctive characteristics of the case study compared to other experimental designs is the use of multiple sources of evidence." Furthermore, there is no way to ensure that the case has been scrutinized when using a single source of evidence, no matter how thorough it might be, and those procedures may be considered pilot research instead of case studies (Gil et al., 2005). 
Gil et al. (2005) arrive at a result similar to this study regarding most case studies' use of few sources of evidence. A possible explanation for this type of problem may be ignorance of the need to ensure data reliability through triangulation, which is discussed in the next criterion. Perhaps importance is also not attributed to the objective of triangulation by the authors, which minimizes the importance of data collection from multiple sources. "The process of triangulation will ensure that discoveries in a case study will be convincing and accurate" according to Martins (2006, p. 80).

Criterion 8 assessed whether there is triangulation of data in the studies reporting the use of multiple sources of evidence. Only 27 papers (15\% of the total) reported using data triangulation, that is, only 27 studies ( $25 \%$ of 105 papers) with multiple sources of evidence used this resource. Cesar et al. (2010) identifies a 36\% frequency of this criterion, a higher value than found in this study. This finding suggests that using multiple sources of evidence may be used for purposes other than data triangulation.

An explanation for this would be the authors' possible lack of awareness regarding the precautions to be taken during data collection on a case study. Another reason may be that many papers (53\%) used the case study as a data collection method and not as a research strategy, as discussed below, which would rule out the use of data triangulation.

Criterion 9 examined whether operational measures were disclosed for the variables analyzed, when necessary, which helps to impart construct validity. The disclosure of operational measures was found in 162 papers (90\%). This criterion showed some methodological rigor and its importance results from the establishment of operational measures appropriate for the concepts studied, according to Yin (2010). The results from similar studies may only be added if the concepts studied are also the same, which helps to assess the study contribution.

Criterion 10 analyzed whether there is an explanation for the data collection method, including the steps followed, when they occurred, how they occurred, with whom they occurred, and in what way they occurred, which also contributes to data reliability. Only $53 \mathrm{pa}-$ pers $(29 \%)$ reported all this information. The number of interviewees was reported in many studies; however, those studies then failed to include other information on those interviewees or the interview method. In Cesar et al. (2010), 95\% of the papers reported the data collection method and $61 \%$ included information on the respondents, the data, and the situations examined in the analysis and according to those authors, that number was expected because it is a formal requirement to be met for submitting papers for publication in the annals of conferences or in journals.

The journals used as data sources in this study have the highest Capes scores. Therefore, it was expected that in their assessments, the reviewers would demand this information be provided in detail. Thus, the explanation for this fact may be (once again) the lack of knowledge about the case study research strategy. Intriguingly, the authors have this information yet fail to detail it in the methods, which may suggest that they do not consider this information key for understanding the research or for data reliability.

Approximately 50 papers (28\%) did not even report the case study methodology and data collection method. The analyses in case studies are based on the data collected; thus, information on the process is necessary both to assist in the study reliability and to enable other interested authors to use it as a basis for further research or possible comparisons among results.

Criterion 11 analyzed the existence of some report or indication regarding the research protocol, which may offer the possibility to replicate the data collection method in other studies. Only nine papers (5\%) reported that information. It is noteworthy that information on the protocol is not mandatory in case studies, although detailed information on the data collection method, which is protocol information, is critical to data reliability.

The analysis of the criteria for the data collection category indicated the existence of confusion among authors between the case study as a research strategy and as a data collection method, as reported by Yin (2010) and Martins (2008). This is further evident when noting that the case study was treated as a data collection method in 96 papers (53\%) because it essentially served to apply the concepts discussed in the literature review section to the company examined, that is, the study applied some model, structure, system, or evaluation, among others, with data collected from the company examined, and it did not examine what the company does or has in a specific situation.

The case study is not and should not be merely a data collection method, as discussed in the theoretical framework. It is a research strategy with specific characteristics and generates satisfactory results regarding the study object only when used correctly. This result indicates that the case study strategy is apparently not well understood by those who work in the scientific production process.

Other interesting facts were observed in this category. One paper evaluated did not even report either the objective or information on data collection. Another paper reported the use of a survey to collect data for the case study. One paper used the literature review as a data source and considered it as a case study. It is also noteworthy that most of the papers that used the case study cited Yin to explain their suitability in the proposed study. However, our analysis seems to suggest that authors cite their explanations out of context.

For example, one of Yin's statements that is most frequently cited in the papers is that "the case study research method is an empirical inquiry that investigates a contemporary phenomenon within its real-life context". This statement is apparently understood by many au- 
thors as the definition of a case study and if analyzed in the absence of an interaction with the other characteristics of the method, it may lead to an understanding that mere data collection in a company may characterize it. Accordingly, further attention is required when explaining the choice of a case study to report other underlying characteristics of this research strategy to ensure the reliability of the results.

Although excluded from the criteria examined, it was found that with respect to data collection, 12 papers (7\%) used interviewees' statements to support the study analyses. This type of evidence could be better used by the authors because transcribing the statements of interviewees helps to support the authors' analyses, especially with respect to critical issues discussed in the study.

Criteria 12 and 13 refer to the data analysis category. Criterion 12 assessed whether there is an explanation for the method used to perform the analysis that helps to provide internal validity. An explanation for the data analysis method was reported in 55 papers $(31 \%)$. The analysis is usually based on comparing the data with the concepts or theories used in the literature review. Most of the papers failed to report any information on the data analysis method, compromising the internal validity, and this is a key issue in qualitative research studies, as discussed by Ahrens and Chapman (2006).

Gil et al. (2005) also find that most of the case studies evaluated lacked clarity with respect to their analytical procedures, and one explanation is that in most cases described only the data that were found. This is also apparently the reason for the lack of a detailed data analysis, based on what has been assessed in this study. Some papers did not use a literature review upon which to base or compare their analysis, only a mere data description. The fact that most papers are descriptive and exploratory may be an explanation for this phenomenon.

The use of content analysis not for data treatment but for data analysis was cited in some papers, which shows the occurrence of confusion in this regard. Content analysis is a set of methods to analyze communications, and according to Bardin (1977), it aims to describe, infer, and interpret the content of statements. Therefore, content analysis is not a data analysis method with respect to the study objective; instead, it is a method used to extract data from the statements that will be used in the analyses.

Criterion 13 analyzed whether the use of theory ( $\sin$ gle case) or replication (multiple cases) as an analytical base occurred when performing a deductive study, which also helps to provide the characteristic of external validity. It is noteworthy that the use of the deductive method was reported in three papers (2\%), all of which used concepts as an analytical base: the use of either theory or replication was not found in these papers.

The case study provides a vehicle through which theories may be generated or modified based on data and is most appropriate where existing theories are inadequate or incomplete or explain only part of the phenomenon of interest, according to Otley and Berry (1994). Cepeda and Martin (2005) also discuss this issue. Thus, it was expected that the results of the analysis would provide more evidence of the use of theories, along with possible changes or even the generation of theories. In contrast, we assessed the use of concepts with no intent to change them or generate new concepts.

When analyzing all types of approaches (except for the deductive approach), only 13 papers (7\%) used replication, $15(8 \%)$ used theory, and $152(84 \%)$ - the majority - used concepts as analytical base, including variable costing, financial statement analysis, real options, local productive arrangements, productive clusters, liquidity and credit risk, and cost/volume/profit ratio, among others. That is, the primary concern was to test the use of concepts in companies, not to study its effect or how it occurs. Those cases indicated that some authors have contact with a concept and decide to apply it to an organization. There is nothing wrong with doing so; the problem is that it is usually done without a research plan, which may lead to a mistakenly chosen method and failure to generate contributions to knowledge.

To complete the analysis of this criterion, a comparison was performed among the 15 papers using theory from the case study and the other criteria evaluated. The results showed that no significant change in the frequency of criteria 1 through 6 (referring to the study object) occurred in those 15 papers. However, the frequency of the criteria related to data collection, data analysis, and results increased.

With respect to data collection, the mean frequency of the five criteria evaluated in this group (from 7 to 11) increased from 39\% to 59\%: that is, in general, the increase rate was approximately $51 \%$. Standing alone, criterion 7 (whether multiple sources of evidence were used) increased from $58 \%$ (for the 180 papers) to $87 \%$ (for the 15 papers); criterion 8 (whether there was triangulation of data) increased from $15 \%$ to $40 \%$; criterion 9 (disclosure of operational measures) increased from $90 \%$ to $100 \%$; criterion 10 (explanation of the data collection method) increased from $29 \%$ to $60 \%$; and criterion 11 (description of the research protocol) increased from $5 \%$ to $7 \%$.

With respect to the data analysis, the frequency of criterion 12 increased from $31 \%$ to $53 \%$, that is, the papers using theory explained the data analysis method at a higher frequency. With respect to the results (criteria 14 and 15), the mean frequency increased from $29 \%$ to $40 \%$. Criterion 14, which shows the paper contributions, increased from $16 \%$ to $27 \%$. Criterion 15 , which involves information about the need to continue the research, increased from $42 \%$ to $53 \%$. This analysis shows that the papers using theory to base the analyses and the results provided more detail about the data collection, data analysis, and the disclosure of results than those papers using concepts or replication.

Criteria 14 and 15 refer to the results category. Criterion 14 assessed whether contributions in knowledge generation compared to previous studies were reported. It was found that 28 papers (16\%) presented this informa- 
tion and compared their results to previous studies, that is, most case studies analyzed failed to highlight their contribution to the literature or practice of management accounting. On this matter, Otley and Berry (1994) argue that researchers using the case study method must clearly state their initial theoretical stances and interpret their results to indicate the theoretical modification triggered by the empirical observation. This shows the importance of situating the case study's contribution to enable adding it to the knowledge already available, that is, the research should have practical relevance (Meer-Kooistra \& Vosselman, 2012; Bogt \& Helden, 2012).

Cesar et al. (2010) do not assess the contributions generated by the studies examined, although they note that only $6 \%$ of the papers attempted to refute the knowledge generated. According to the authors, this incidence shows that the researchers did not attempt to refute the knowledge generated, nor did they consider it important to include any comments on the subject in their papers.

In this study, the percentage of papers that explained their contributions suggests that researchers conduct their studies separately from the literature, without analyzing what has already been reported and what has been discovered on the subject examined. However, the reason for drafting a scientific paper is its contribution to the field. Another possible explanation may be the fact that as discussed above, most of the studies analyzed used the case study data to test models or concepts.

The criticism of this study proposal is that it is inadequate to assess whether the model/concept may be calculated or implemented in the company: instead, what matters is to discover in companies that use or have used that model/concept why it worked, and/or what worked, and what did not work and/or why. This information contributes to the literature on the subject and helps other companies that have implemented or intend to implement that cost management tool or other model/ concept.

Criterion 15 analyzed whether the study warned about issues that need further research. This information was found in 76 papers (42\%), along with recommendations for future studies. Cesar et al. (2010) find this characteristic in $39 \%$ of papers; the disclosure that the research generated grounds for further research was not observed in any study, but the authors do not discuss the reasons for this finding. Most of the studies were expected to show this type of information because case studies usually approach specific issues and analyze them in depth. Therefore, the existence of untested variables or theories that could complement the findings is usually emphasized as an issue requiring further research. This result may be explained by the fact that most studies are descriptive or exploratory and have primarily used the case study strategy as a data collection method.

Because of the importance of highlighting the research results as a way of showing the contributions of the papers, an analysis was performed comparing the data from the papers that contained the two criteria comprising this group (14 and 15) with the other criteria evaluated to assess whether differences occurred in the frequencies. Fifteen papers were identified that both explained their contributions and warned about issues requiring further research ( $8 \%$ of the total), and no significant differences in the frequencies were found between these 15 papers and the 180 papers in the sample.

That finding is noteworthy because papers highlighting their contributions and warning about issues requiring further research were expected to validate their findings by showing more rigor with respect to the other criteria than the other papers. This suggests that even when papers highlight their contributions, they may have issues with methodological rigor that compromise the validity and reliability of their results.

There was no specific criterion addressing external validity; therefore, inferences could not be made in that regard. However, Alves-Mazzotti (2006, p. 648) argues, "Researchers may offer the opportunity for vicarious experiences through a dense and vivid narrative, that is, they may lead readers to associate what was observed in that case to events experienced by them in other contexts". Accordingly, the issue of narrative written by the author may be the crucial issue in the search for methodological rigor, that is, the detailed description and the presence of transparent information related to these criteria.

\subsection{Summary and Comparison of Results.}

After completing the data analysis, a summary (outlined in Table 4) was performed that included the research criteria, the respective observed frequencies, and results from the study by Cesar et al. (2010) that could be compared to the results from this study. 


\begin{tabular}{|c|c|c|c|c|c|c|}
\hline \multicolumn{2}{|l|}{ Categories } & Criteria & \multirow{2}{*}{$\begin{array}{c}\begin{array}{c}\text { Absolute } \\
\text { Frequency }\end{array} \\
39\end{array}$} & \multirow[t]{2}{*}{$\begin{array}{l}\text { Relative } \\
\text { Frequency }\end{array}$} & \multirow{2}{*}{$\begin{array}{c}\text { Observations } \\
22 \%\end{array}$} & \multirow[t]{2}{*}{$\begin{array}{l}\text { Results from } \\
\text { Cesar et al. } \\
(2010)\end{array}$} \\
\hline \multirow{6}{*}{$\begin{array}{l}\text { The study } \\
\text { object }\end{array}$} & 1 & $\begin{array}{l}\text { Does the study seek to understand a phenomenon in its real-life } \\
\text { context? }\end{array}$ & & & & \\
\hline & 2 & Was the reason for the choice of the case study strategy explained? & 23 & & $13 \%$ & $100 \%$ \\
\hline & 3 & $\begin{array}{l}\text { Is there a connection between phenomenon and context at any stage } \\
\text { of the research? }\end{array}$ & 149 & & $83 \%$ & $\begin{array}{l}\text { There was no } \\
\text { concern about the } \\
\text { criterion }\end{array}$ \\
\hline & 4 & What type of question is raised in the research? & & & $\begin{array}{l}63 \%(113) \\
\text { included no } \\
\text { questions }\end{array}$ & \\
\hline & 5 & What is the case study type? & & & $\begin{array}{l}\text { Most are } \\
\text { exploratory or } \\
\text { descriptive }\end{array}$ & \\
\hline & 6 & Is the case analyzed representative of the study objective? & 51 & $28 \%$ & & \\
\hline \multirow{4}{*}{$\begin{array}{l}\text { The data } \\
\text { collection }\end{array}$} & 8 & Is there data triangulation among the sources of evidence? & 27 & $15 \%$ & & $36 \%$ \\
\hline & 9 & $\begin{array}{l}\text { Were operational measures regarding the variables analyzed } \\
\text { disclosed, when appropriate? }\end{array}$ & 162 & $90 \%$ & & \\
\hline & 10 & $\begin{array}{l}\text { Is there an explanation for the data collection method, including } \\
\text { the steps followed, when they occurred, where they occurred, with } \\
\text { whom, and in what way? }\end{array}$ & 53 & $29 \%$ & & $61 \%$ \\
\hline & 11 & Is there any report or evidence about the research protocol? & 9 & $5 \%$ & & \\
\hline \multirow{2}{*}{$\begin{array}{l}\text { The data } \\
\text { analysis }\end{array}$} & 12 & Is there an explanation of how the analyses were conducted? & 55 & $31 \%$ & & \\
\hline & 13 & $\begin{array}{l}\text { Were theory (single-case study) or replication (multiple-case study) } \\
\text { used as a basis for the analysis when conducting deductive studies? }\end{array}$ & & & $\begin{array}{c}\text { Most }(84 \%) \\
\text { used concepts }\end{array}$ & \\
\hline \multirow[t]{2}{*}{ The results } & 14 & $\begin{array}{l}\text { Were contributions to knowledge generation reported in terms of } \\
\text { comparisons to previous studies? }\end{array}$ & 28 & $16 \%$ & & $\begin{array}{l}6 \% \text { refuted the } \\
\text { knowledge } \\
\text { generated }\end{array}$ \\
\hline & 15 & Does the study warn about issues requiring further research? & 76 & $42 \%$ & & $39 \%$ \\
\hline
\end{tabular}

The critical aspects concerning the results were the following: (i) little emphasis on explaining the need to understand the phenomenon in its context (22\%); (ii) lack of explanation for the purpose of choosing the case study strategy (13\%); (iii) predominant use of questions that preclude deepening the analyses; (iv) few studies of the explicative or explanatory type (only $5 \%$ ), which may enable deeper results; (v) lack of a rationale for the choice of single- or multiple-case study; (vi) the use of only one source of evidence in many studies (42\%); (vii) little use of triangulation of data and information (15\% of papers); (viii) little emphasis on information about the data collection method (29\%); (ix) little reference to the use of the research protocol or details related to data collection; (x) confusion between the case study as a research strategy and as a data collection method in a high number of cases (53\%); (xi) a low number of papers explaining the data analysis method (31\%); (xii) greater emphasis on the use of concepts than on the use of theories or replication; (xiii) few reports of the contributions generated by the study, especially compared to previous studies (16\%); and (xiv) failure to highlight the issues requiring further research (42\%).

\section{CONCLUSIONS AND RECOMMENDATIONS}

This study examines the methodological rigor of management accounting case studies published in Brazil. Based on the analysis of those studies' results, it is concluded that in several aspects, case studies in management accounting have no methodological rigor. This panorama highlights the gap between how a case study should be conducted and how researchers are using this resource in practice and identifies potential opportunities for improvement.
The profile of the papers included in the sample examined focuses on data description instead of explanation, on the use of concepts instead of theories, with insufficient detailing of how the study was conducted and how the analyses were performed, thereby generating isolated results, and only $16 \%$ of the papers reported contributions compared to previous studies. This profile provided evidence to support the notion that several methodological rigor criteria had frequency rates closer to $0 \%$ than $100 \%$. 
One finding that triggered evidence in that regard was that most of the papers analyzed used descriptive and exploratory types of study. These types of study usually do not aim to explain the results, only to describe the data. Therefore, they tend to provide less detail than the case study process, thus showing lower rigor. These results are consistent with Zimmerman's (2001) argument that the empirical literature on management accounting is focused on reporting company practices. Descriptive studies per se neither advance the literature nor create an understanding of management accounting practices in a coherent manner (Zimmermann, 2001). Most of the studies were observed to have isolated data, with no link to similar studies or even theories that might explain the findings derived from the data.

This observation was confirmed when noting that studies that used theories to support their results had higher methodological rigor rates than the papers using concepts, especially for criteria related to data collection, data analysis, and results. This finding supports the notion that explanatory-type papers or papers that use theoretical bases to support the results provide more detail about the information related to methodological rigor. Another reason that may be linked to the performance of various criteria is that most of the papers (53\%) used the case study as a method of data collection, not as a research strategy. This leads to a distortion of the strategy because it does not comply with the strategy's requirements and it compromises the results. Another factor that may explain the findings is that the authors may not have considered it important to provide detailed information - or they may even have been unaware of this requirement.

Cesar et al. (2010) obtains different results related to the percentages of items that can be compared with the present study, indicating that no improvement occurred. In contrast, the items were less frequent in this study, except for criteria 15 , with respect to the need to continue the research. Other studies corroborate the results found, including Gil et al. (2005) and Consoli et al. (2008).

Based on the findings, the issues indicated by scholars in the field discussed in the literature review were detected in the sample examined. This shows the need to understand the case study strategy, analyzing its fundamental principles and applying them to take advantage of its potential; moreover, it indicates that the issues raised about the case study are more closely related to how it has been used than to the method itself.

One of this study's contributions was its presentation of the key issues requiring attention from researchers using the case study approach, to increase the methodological rigor of case studies. This was achieved by introducing a set of guidelines for conducting case studies and how to apply them as quality criteria to assess their methodological rigor.

Another contribution was the identification of the gap between what is proposed as a case study according to the literature and what was implemented in management accounting studies in Brazil, which demonstrates the need to make an effort to change this situation to produce quality studies with reliable results. The importance of management accounting studies lies in the possibility of their results to help the practice (Meer-Kooistra \& Vosselman, 2012) and one way to reach this goal is to make adequate use of the strategies available for its implementation.

It is recommended that future studies examine other possible reasons that might explain this study's findings, using authors, professors, reviewers, and other people involved in the process of producing and publishing research on management accounting case studies. Other studies should also focus on investigating the specific content of papers that use case studies as research strategy to assess, for example, whether studies claiming to be explanatory may actually be regarded as such. 


\section{References}

Ahrens, T., \& Chapman, C. (2006). Doing qualitative field research in management accounting: positioning data to contribute to theory. Accounting, Organizations \& society, 31(8), 819-841.

Alves-Mazzotti, A. J. (2006). Usos e abusos dos estudos de caso. Cadernos de Pesquisa, 36(129), 637-651.

Bardin, L. (1977). Análise de conteúdo. São Paulo: Persona.

Berry, A. J., \& Otley, D. (2004). Case-based research in accounting. In C. Humphrey, \& B. Lee, The real life guide to accounting research: a behind-the-scenes view of using qualitative research methods (pp. 231-255). Oxford: Elsevier.

Bogt, H. T., \& Helden, J. V. (2012). The practical relevance of management accounting research and the role of qualitative methods therein: the debate continues. Qualitative Research in Accounting \& Management, 9(3), 265-295.

Cepeda, G., \& Martin, D. (2005). A review of case studies publishing in Management Decision 2003-2004: guides and criteria for achieving quality in qualitative research. Management Decision; 43(6), 851-876.

Cesar, A. M. R., Antunes, M. T. P., \& Vidal, P. G. (2008). A utilizaçẩo do método do estudo de caso em pesquisas das áreas de operações, recursos humanos e contabilidade. In Anais do $32^{\circ}$ Encontro da ANPAD. Rio de Janeiro, RJ.

Cesar, A. M. R., Antunes, M. T. P., \& Vidal, P. G. (2010). Método do estudo de caso em pesquisas da área de contabilidade: uma comparação do seu rigor metodológico em publicações nacionais e internacionais. Revista de Informação Contábil, 4(4), 42-64.

Chua, W. F. (1986). Radical developments in accounting thought. The Accounting Review, 61(4), 601-632.

Consoli, M. A., Musetti, M. A., Scare, R. F., \& Fratantonio, W. A. (2008). Uma discussão sobre a utilização do estudo de casos como método de pesquisa em ciências gerenciais. In Anais do $32^{\circ}$ Encontro da ANPAD. Rio de Janeiro, RJ.

Cooper, D. J., \& Morgan, W. (2008). Case study research in accounting. Accounting Horizons, 22(2), 159-178.

Eisenhardt, K. M. (1989). Building theories from case study research. The Academy of Management Review, 14(4), 532-550.

Gil, A. C., Licht, R. H. G., \& Oliva, E. C. (2005). A utilização de estudos de caso na pesquisa em administração. Revista de Administração e Contabilidade da Unissinos, 2(1), 47-56.

Godoy, A. S. (2006). Estudo de caso qualitativo. In C. K. Godoi, R. B. S. Melo, \& A. B Silva (Org.), Pesquisa qualitativa em estudos organizacionais: paradigmas, estratégias e métodos (pp. 115-146). São Paulo: Saraiva.

Hägg I., \& Hedlund G. (1979). "Case studies” in accounting research.
Accounting, Organizations and Society, 4(1/2), 135-143.

Lee, B., Collier, P. M., \& Cullen, J. (2007). Reflections on the use of case studies in the accounting, management and organizational disciplines. Qualitative Research in Organizations and Management: An International Journal, 2(3), 169-178.

Lillis A. M., \& Mundy J. (2005). Cross-sectional field studies in management accounting research: closing the gaps between surveys and case studies. Journal of Management Accounting Research, 17, 119-141.

Lima, J. P. C., Antunes, M. T. P., Mendonça Neto, O. R., \& Peleias, I. R. (2012). Estudos de caso e sua aplicação: proposta de um esquema teórico para pesquisas no campo da contabilidade. Revista de Contabilidade e Organizações, 6(14), 127-144.

Martins, G. A. (2006). Estudo de caso: uma estratégia de pesquisa. São Paulo: Atlas.

Martins, G. A. (2008). Estudo de caso: uma reflexão sobre a aplicabilidade em pesquisas no Brasil. Revista de Contabilidade e Organizações, 2(2), 8-18.

Martiṇs, G. A., \& Theóphilo, C. R. (2007). Metodologia da investigação científica para ciências sociais aplicadas. São Paulo: Atlas.

Meer-Kooistra, J. V., \& Vosselman, E. (2012). Research paradigms, theoretical pluralism and the practical relevance of management accounting knowledge. Qualitative Research in Accounting \& Management, 9(3), 245-264.

Merriam, S. B. (2009). Qualitative research: a guide to design and implementation. San Francisco: Jossey-Bass.

Otley, D. T., \& Berry, A. J. (1994). Case study research in management accounting and control. Management Accounting Research, 5(1), 45-65.

Scapens, R. W. (1990). Researching management accounting practice: the role of case study methods. British Accounting Review, 22, 259-281.

Scapens, R. W. (2004). Doing case study research. In C. Humphrey, $\& \mathrm{~B}$. Lee, The real life guide to accounting research: a behind-thescenes view of using qualitative research methods (pp. 257-279). Oxford: Elsevier.

Yin, R. K. (1981). The case study crisis: some answers. Administrative Science Quarterly, 26(1), 58-65.

Yin, R. K. (2010). Estudo de caso: planejamento e métodos (4a ed.). Porto Alegre: Bookman.

Zimmerman, J. L. (2001). Conjectures regarding empirical managerial accounting research. Journal of Accounting and Economics, 32, $411-427$ 